\title{
The Nursing Research of Frozen Fresh Potato Topical Treatment Infusion Leakage
}

\author{
Fenge Zhang ${ }^{1}$, Weirong Jin ${ }^{2,}$, Yan Wang ${ }^{3}$ \\ ${ }^{1}$ Department of Emergency, Liaocheng People's Hospital, Liaocheng, China \\ ${ }^{2}$ Department of Formulation Branch, Liaocheng People's Hospital, Liaocheng, China \\ ${ }^{3}$ Department of Pharmacy, Yantai Traditional Chinese Medical Science Hospital, Yantai, China
}

Email address:

lcsrmyyjwr@163.com (Weirong Jin)

${ }^{*}$ Corresponding author

\section{To cite this article:}

Fenge Zhang, Weirong Jin, Yan Wang. The Nursing Research of Frozen Fresh Potato Topical Treatment Infusion Leakage. American Journal of Nursing Science. Vol. 7, No. 4, 2018, pp. 143-146. doi: 10.11648/j.ajns.20180704.15

Received: April 13, 2018; Accepted: June 5, 2018; Published: June 29, 2018

\begin{abstract}
Objective: Intravenous (IV) infusions are an essential part of hospital patient care, but Intravenous Infiltration in the process of the treatment. Leakage brought great pain to the patient; So far, no research has been done in this area. A simple and cheap method to relieve pain of IV infusions in this Study. Method: According to the accepted methods of treatment, all patients were divided into three groups randomly, Group A: Wash and dry the fresh potatoes. Group B:Apply the Hirudoid cream clockwise evenly and massage the puncture point within 5 to $8 \mathrm{~cm}$ of the wound area. 20 minutes three times a day. Group C: Saline flushes out and local cold. Patients in the three groups were treated with the same methods. Conclusion: Study shows $96.67 \%$ patient of Group A had recovered from symptoms, of redness and vein streaking dissipated, blood vessels returning to a normal condition. This study provides a method with frozen fresh potato chips in the treatment of symptoms of leakage.
\end{abstract}

Keywords: Nursing Research, Frozen Fresh Potato, Treatment Infusion Leakage

\section{Introduction}

The use of intravenous (IV) access for the provision of nutrition and medication is essential in modern neonatal intensive care. However, accidental leakage of an infusion into the surrounding tissues frequently occurs [1]. In clinical treatment, extravasation is defined as an inadvertent instillation or leakage of cytotoxic drugs into the per vascular space during infusion [2]. Leakage not only brought great pain to the patient, such as the pain, indurations, redness, and limited movement. But also increase medical expenses for treatment, the period of hospitalization . Not only newborn [3], young children [4], but also adult [5] suffering from Leakage.

Potatoes are the fourth most plentiful food crop in the world, yet the scientific literature on the health effects of potato consumption is scarce [6]. The potato is medicine edible plant which recorded in the in the book of Traditional Chinese medicine. It can detoxify detumescence recorded in the book. Potato phenolics impact starch digestion and glucose transport in model systems but translation to phenolic rich potato chips results in only modest modification of glycemic response in humans [7]. Roduct reformulation initiatives have improved existing products for most food group-nutrient pairs. Spiteri M [8] had found the contribution of food reformulation to the change in nutritional quality of food purchases was strong in potato chips. Animal experimental study indicated [9] potato chips external applying could treat intravenous infusion extravasation. So far, they haven't been found the study of fresh potato slices' intervention effect on symptoms caused by infusion extravasation. This study aimed to investigate the nursing research of fresh frozen fresh potato topical treatment infusion leakage.

\section{Methods and Patients}

\subsection{Study Design}

This retrospective study was conducted in Mount Taishan 
medical school affiliated Liaocheng City people's Hospital emergency department between March, 2014 and 31 March, 2017. The protocols were approved by the Hospital Ethics Committee. All patients (or the guardian of a minor) provided written informed consent before enrollment.

\subsection{Inclusion Criteria}

The inclusion criteria were as follows:

Patients with infusion (sugar, salt water as the solvent) leakage, the leakage area $\leq 20 \mathrm{~cm} \times 20 \mathrm{~cm}$. Leakage occurred after 0.5 hours for processing

\subsection{Exclusion Criteria}

The exclusion criteria were as follows:

Patients with infusion(sugar, salt water as the solvent) leakage had to deal with other methods.

The skin lesions were caused by leakage of fluids(sugar, salt water as the solvent).

\subsection{Fresh Potato}

Fresh potato was from annual herb solanaceous tomato potato, which had been identified by Medicine Professor Jun-ying Shi. Frozen fresh potato(in $0-5^{\circ} \mathrm{C}$ refrigerator cold treated more than 3 hours) used is cut into $0.1 \sim 0.3 \mathrm{~cm}$. ( Figure 1 ).

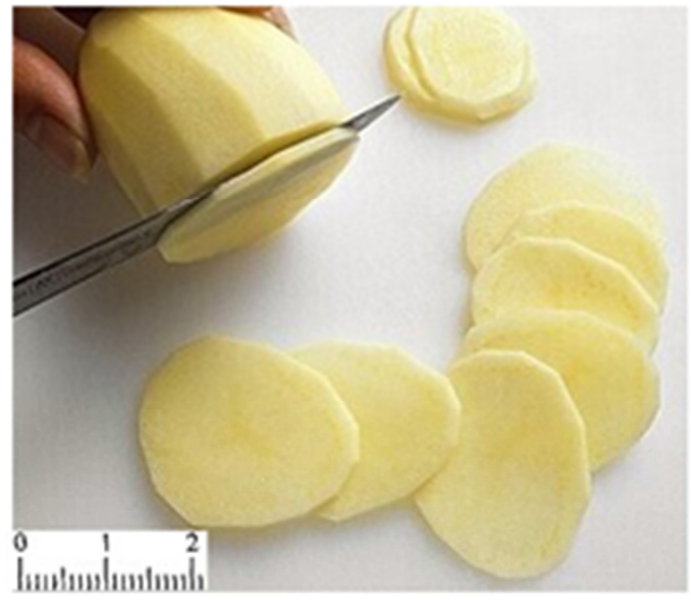

Figure 1. Photo chips.

\subsection{Hirudoid}

The main ingredient of Hirudoid is sulfonate grease mucopolysaccharides (MPS). It can effectively control inflammatory disease, improve the blood circulation of the affected area, absorbs the exudate liquid, and cures the edema. It can be as a control [10].

\subsection{The Evaluation Index [11]}

Full recovery (I degree): Patients had recovered from symptoms, of redness and vein streaking dissipated, blood vessels returning to a normal condition.

Marked improvement(II degree): Pain and swelling has subsided, blood vessels are not as stiff, more flexible.

Effective (III degree): Pain reduced, redness and swelling has subsided, streaking is lighter.

Not effective (IV degree): No visible improvement of symptoms.

\subsection{Groups and Treatment}

According to the accepted methods of treatment, all patients were divided into three group randomly: Fresh potato group (Group A), 30 patients with a mean age of $33.11 \pm 12.25 \mathrm{y}$. Hirudoid group (Group B), 30 patients with a mean age of $33.17 \pm 10.15$ y. Control group (Group C); 30 patients with a mean age of $32.98 \pm 10.12$ y. Extravasations occurred should immediately stop the infusion of medicine. The groups were treated at the same time and for the same duration as follows:

Group A: Wash and dry the fresh potatoes. Cut them in slices 5 to $8 \mathrm{~cm}$ in diameter and $0.1 \sim 0.3 \mathrm{~cm}$ thick. Apply the slices around the wound and avoid the puncture point. Stabilize the slices with plastic wrap.

Group B: Apply the Hirudoid cream clockwise evenly and massage the puncture point within 5 to $8 \mathrm{~cm}$ of the wound area. 20minutes three times a day.

Group C: Saline flushes out and local cold.

All patients of three groups of patients were not statistically significantly different in gender, age, continuing medication time $(\mathrm{P}>0.05)$.

\subsection{Statistical Analysis [12]}

All data were analyzed by one-analysis of variance, and the differences between means were established by Duncan's multiple-range test. Multiple sets of curative effect comparison $\chi^{2}$ test. Data represent means and standard deviations. $\mathrm{P}<0.05$ was considered to indicate statistical significance.

\section{Outcomes}

Demographic characteristics and baseline measurements

This study is a retrospective study. Among the 90 remaining eligible patients, there were no significant differences between the groups in terms of demographic characteristics and leakage area (Table 1).

Table 1. Patient grouping.

\begin{tabular}{lllll}
\hline \multirow{2}{*}{ Group } & Sex & \multirow{2}{*}{ Age } & Leakage area $\left(\mathbf{c m}^{2}\right)$ \\
\cline { 2 - 4 } & (Male/Female) & Length $\mathbf{x}$ width & \multicolumn{2}{c}{ Mean \pm SD } \\
\hline $\mathrm{A}$ & $13 / 17$ & $43.20 \pm 12.22$ & $18.00 \times 15.00$ & $270.01 \pm 1.32$ \\
$\mathrm{~B}$ & $14 / 16$ & $43.18 \pm 10.13$ & $19.80 \times 14.05$ & $278.14 \pm 0.91$ \\
$\mathrm{C}$ & $15 / 15$ & $42.96 \pm 10.09$ & $18.01 \times 16.00$ & $288.16 \pm 2.01$ \\
${ }^{*} \mathrm{P}$ & & 0.9955 & & 0.1010 \\
\hline
\end{tabular}

$* P>0.05$ (among the three groups) 
Different degrees of efficacy were observed among the groups after treatment for 3, 5, and 10 days. Compared to the $\mathrm{C}$ groups, significantly greater clinical efficacy was observed in the patients treated with Group $\mathrm{A}(P<0.05)$; there were no significant difference between $\mathrm{B}$ and $\mathrm{A}(\mathrm{P}>0.05)$; But potatoes was much more cheaper than Hirudoid cream, and are easier to get than Hirudoid cream (Group A) (Table 2).

Table 2. Effect of three groups.

\begin{tabular}{|c|c|c|c|c|c|c|c|c|c|}
\hline Group & $\mathbf{A}$ & & & B & & & $\mathbf{C}$ & & \\
\hline Cases & 30 & & & 30 & & & 30 & & \\
\hline Degree & I & II & III & $\mathrm{I}$ & II & III & I & II & III \\
\hline 3 days of significant effect & 22 & 6 & 2 & 18 & 7 & 5 & 8 & 6 & 16 \\
\hline 5 days of significant effect & 28 & 1 & 1 & 20 & 5 & 5 & 8 & 8 & 14 \\
\hline 7 days of significant effect & 29 & 1 & 0 & 21 & 5 & 4 & 8 & 8 & 14 \\
\hline Compare & $* \mathrm{P}$ & & & ${ }^{\Delta} \mathrm{P}$ & & & $* \mathrm{P}$ & & \\
\hline
\end{tabular}

A compare $\mathrm{C}: * \mathrm{P}<0.05$; A compare $\mathrm{B}:{ }^{\wedge} \mathrm{P}>0.05$

\section{Discussion}

Study shows $96.67 \%$ patient of Group A had recovered from symptoms, of redness and vein streaking dissipated, blood vessels returning to normal condition. But Group C had only $36.67 \%$. Group A had a significant difference compared with group $\mathrm{C}$, but compared with group $\mathrm{B}$, no difference. This study to prove the fresh frozen potato chips in the treatment of symptoms of leakage. Potato chips, as a kind of medicinal and edible plant, are effective, economical, and easy to be accepted and operated by the patient's family members, which can reduce the patient's pain $[13,14]$. It will be widely used in treating infusion leakage

\section{Conclusion}

Although regular training and education, which are best provided by a specialised and experienced task force including all disciplines involved in therapy, once the infusion leakage occurs. However, once it has been detected, emergency measures must be taken immediately. Nurses should he more observant and familiarize themselves with the effects administration and side effects of the condition so as to properly assess the condition and take appropriate care. In addition [15], some scholars have found that of Glycosides in potatoes have anti-inflammatory effects on the stimulation of macrophages and prophages in mice. In this study, the interventions taken by the experimental group greatly reduce the occurrence of phlebitis, reducing the suffering of the patients, and should therefore be considered for clinical applications.

\section{Conflict of Interest}

The authors declare no financial or other conflicts of interest.

\section{References}

[1] An B, Ning H. Accidental infusion leakage at subgalea in infants: report of 6 cases. Int J Clin Exp Med. 2015 Aug 15; 8(8):11943-6.
[2] Ursula Pluschnig. Werner Haslik. Rupert Bartsch. etc. Extravasation emergencies: state-of-the-art management and progress in clinical research. memo (2016) 9:226-230.

[3] Wade S Kingery, Tian-Zhi Guo, Geeta S Agashe, M. Frances Davies, J. David Clark, Mervyn Maze. Glucocorticoid inhibition of neuropathic limb edema and cutaneous neurogenic extravasation [J]. Brain Research, 2001, 913(2).

[4] T. Loeb, F. Jegou, P. Lemarchand, M. Pinsard, M. Canonne. Ischémie de membre par extravasation d'une perfusion veineuse périphérique d'adrénaline $[\mathrm{J}]$. Reanimation Urgences, 1995, 4(6).

[5] [1]Wade S Kingery, Tian-Zhi Guo, Geeta S Agashe, M. Frances Davies, J. David Clark, Mervyn Maze. Glucocorticoid inhibition of neuropathic limb edema and cutaneous neurogenic extravasation [J]. Brain Research, 2001, 913(2).

[6] Åsli LA1, Olsen A2, Braaten T, etc. Potato Consumption and Risk of Colorectal Cancer in the Norwegian Women and Cancer Cohort. Nutr Cancer. 2017 Mar 21:1-9.

[7] Moser S1, Aragon I1, Furrer A1, etc. Potato phenolics impact starch digestion and glucose transport in model systems but translation to phenolic rich potato chips results in only modest modification of glycemic response in humans. Nutr Res. 2018 Feb 12. pii: S0271-5317(17)30845-X. Rong Xiaoshan.

[8] Peng Youqing, Wei Youzhen. Animal experimental study on potato chips external applying to treat intravenous infusion extravasation. Chinese Nursing Research March, 2015 Vo1. 29 No. 3A:791-793.

[9] Rong Xiaoshan, Peng Youqing, Wei Youzhen. Animal experimental study on potato chips external applying to treat intravenous infusion extravasation. CHINESE NURSINU RESEARCH. March, 2015 Vo1. 29 No. 3A:791-793.

[10] Spiteri M1, Soler LG2. Food reformulation and nutritional quality of food consumption: an analysis based on households panel data in France. Eur J Clin Nutr. 2018 Feb;72(2):228-235.

[11] LI Jing-hong, GAN Mi-mi. Comparison between two methods of preventing Amiodarone induced phlebitis. South China JourncrL of Cardiology. 2014 Sep 15:198-202.

[12] Jin Wei-rong, Zhang Feng-e, Diao Bao-zhong, etc. Clinical outcomes of Wulingsan subtraction decoction treatment of postoperative brain edema and fever as a complication. Evidence-Based Complementary and Alternative Medicine, vol. 2016, Article ID 5078689. Liuyuhan, Xuejunbo, Houleilei. 
[13] Rong Xiaoshan, Peng Youqing, Wei Youzhen. Animal experimental study on potato chips external applying to treat intravenous infusion extravasation. Nursing research, 2015, 29(07):791-793.

[14] Application of raw potato chips in the extravasation of mannitol infusion. Chin J Misdiagn, Feb 2009 Vol 9 No. 5. 1252.
[15] Olivia M. Kenny, Catherine M. McCarthy, Nigel P. Brunton, Mohammad B. Hossain, Dilip K. Rai, Stuart G. Collins, Peter W. Jones, Anita R. Maguire, Nora M. O'Brien. Anti-inflammatory properties of potato glycoalkaloids in stimulated Jurkat and Raw 264.7 mouse macrophages [J] 2013, Life Sciences(13): 775-82. 